\title{
Perinatal outcomes of two consecutive strategies for the management of fetal growth restriction: a before-after study
}

Sabrina Paola Demirdjian ( $\sim$ sdemirdj@cas.austral.edu.ar)

Hospital Universitario Austral https://orcid.org/0000-0001-7675-2100

Cesar Hernan Meller

Maria Celeste Berruet

Gonzalo Dosdoglirian

Adolfo Etchegaray

\section{Research Article}

Keywords: fetal growth restriction, placental insufficiency, Doppler assessment, neonatal morbidity, respiratory distress syndrome, low birthweight.

Posted Date: March 2nd, 2022

DOI: https://doi.org/10.21203/rs.3.rs-1393751/v1

License: () (1) This work is licensed under a Creative Commons Attribution 4.0 International License.

Read Full License 


\title{
Perinatal outcomes of two consecutive strategies for the management of fetal growth restriction: a before-after study
}

Authors: Sabrina Paola Demirdjian MD MSc, Cesar Hernan Meller MD MSc, Maria Celeste Berruet MD, Gonzalo Dosdoglirian MD, Adolfo Etchegaray MD

Corresponding author: Sabrina Paola Demirdjian sdemirdj@cas.austral.edu.ar

\begin{abstract}
Purpose We aim to compare the perinatal outcomes of two consecutive management strategies for fetal growth restriction (FGR), with or without the inclusion of additional Doppler parameters.
\end{abstract}

Methods A quasi-experimental before/after study was conducted in which we compared a composite perinatal outcome, prematurity rate and neonatal complications between two management strategies in small fetuses. In the strategy 1 (S1), the management was based on fetal biometry and umbilical artery Doppler. The second strategy (S2) added the assessment of uterine and middle cerebral artery Doppler. We also compared outcomes between strategies according to early ( $\leq 32$ weeks) and late ( $>32$ weeks) diagnosis subgroups.

Results We included 396 patients, 163 in S1 and 233 in S2. There were no significant differences in the perinatal composite outcome ( $\mathrm{p}$ 0.98), prematurity ( $\mathrm{p}$ 0.19) or in the subgroup analysis. We found a significant reduction in respiratory distress syndrome (RDS) rate with S2 both globally (OR 0.50, p 0.02), and in the early diagnosis subgroup (OR 0.45, p 0.01). In addition, we observed a significant reduction in the incidence of sepsis with S2 both globally (OR 0.30, p 0.04) and in the early diagnosis subgroup (OR 0.25, p 0.02). We did not observe significant differences in necrotizing enterocolitis ( $\mathrm{p} 0.41)$ and intraventricular hemorrhage (p 1.00).

Conclusion The expanded strategy for the management of FGR did not show significant differences in the primary composite outcome or prematurity. However, it was associated with a lower incidence of RDS and neonatal sepsis.

Keywords fetal growth restriction; placental insufficiency; Doppler assessment; neonatal morbidity; respiratory distress syndrome; low birthweight.

\section{Introduction}

Fetal growth restriction (FGR) due to placental insufficiency is defined by an estimated fetal weight (EFW) lower than the 10th percentile with Doppler signs of placental insufficiency [1-3]. It is one of the leading causes of perinatal morbidity and mortality, associated with up to $30 \%$ of intrauterine deaths in the third trimester [4]. Recently, it has also been linked to a greater predisposition to pediatric and adult non-communicable diseases such as diabetes mellitus, obesity, high blood pressure and cardiovascular disease [5-7]. 
Although several interventions have been proposed to improve the prognosis of these fetuses, none of them have shown to be effective [8-15]. For this reason, so far, the only available tool to manage these cases is early detection and timely delivery.

Traditionally, screening and management were based exclusively on ultrasound biometry and umbilical artery Doppler [1,16-18]. According to this strategy, fetuses with an EFW below the 10th percentile with normal umbilical artery Doppler were considered to be small for gestational age (SGA), while those with abnormal umbilical artery Doppler were labeled as growth-restricted [19-21]. However, different groups and scientific societies have recently advocated for the introduction of a more comprehensive definition for FGR that includes the evaluation of other Doppler parameters to standardize the obstetric management of this condition [4, 22, 23]. This new definition includes extremely small fetuses (EFW less than the 3rd percentile) and fetuses with EFW between the 3rd and 10th percentile and at least one of the following abnormal Doppler parameters: umbilical artery pulsatility index (UmA-PI) over the 95th percentile, middle cerebral artery pulsatility index (MCA-PI) below the 5th percentile, the ratio between these two vessels (cerebro-placental ratio, CPR) below the 5th percentile and mean pulsatility index of uterine arteries (UtA-PI) over the 95th percentile [24].

The aim of this study was to compare the perinatal outcomes between these two strategies for the management of FGR during two consecutive periods in a single tertiary center.

\section{Methods}

\section{Study Design}

This was a quasi-experimental before/after study in which we compared a perinatal composite outcome between two management strategies in patients with small fetuses followed in our Unit from June 2009 to April 2019.

\section{Groups and definitions}

We included all singleton pregnancies with an EFW less than the 10th percentile and available perinatal outcomes. We excluded all cases with fetal genetic and structural anomalies and prenatal infections.

Patients were classified and managed from June 2009 to July 2014 according to Strategy 1 (S1) (Fig. 1) [18], and from July 2014 to April 2019 according to Strategy 2 (S2) (Fig. 2) [24].

Briefly, in S1, SGA was defined as an EFW below the 10th percentile with normal umbilical artery Doppler and in S2, as an EFW between the 3rd and 10th percentile with normal Doppler parameters (UmA-PI, MCA-PI, CPR, or UtA-PI). In S1, the diagnosis criteria for FGR was an EFW below the 10th percentile with an abnormal umbilical artery Doppler. In S2, the diagnosis of FGR was based on the updated definition proposed in 2014 [24], ie, either an EFW below the 3rd percentile or an EFW between the 3rd and 10th percentile with an abnormal Doppler (UmA-PI, MCA-PI, CPR, or UtA-PI). Each strategy had its own recommended follow-up intervals and gestational ages for delivery according to severity (Fig. 1,2). 
In both strategies, the decision to administer steroids and magnesium sulfate was based on the gestational age (GA) at birth.

\section{Outcomes}

The primary outcome was a composite of perinatal death or the need for mechanical ventilation (NMV) or admission to the Neonatal Intensive Care Unit (NICU).

The secondary outcomes were: incidence of prematurity and associated complications, such as respiratory distress syndrome (RDS), sepsis, intracranial hemorrhage (ICH), and necrotizing enterocolitis (NEC).

We compared the primary and secondary outcomes between strategies according to early ( $\leq 32$ weeks) and late (> 32 weeks) diagnosis [25].

\section{Statistical Analysis}

We analyzed the primary and secondary outcomes as dichotomous variables. We calculated the odds ratios (OR) using a simple logistic regression model to analyze the association between the two strategies and the primary and secondary outcomes. We analyzed separately each component of the composite outcome using independent logistic regression models.

Numerical variables were described with mean and standard deviation or median and interquartile range according to distribution and categorical variables with percentage and absolute frequency. T-test or Mann-Whitney test were used for continuous variables according to distribution and Chi2 or Fisher test for categorical variables.

All data analysis was done using the Stata 13.0 software package.

A p-value of less than 0.05 was considered statistically significant.

The study was approved by the institutional review board (P20-035).

\section{Results}

Among 812 patients, 396 met the inclusion criteria, 163 in S1 and 233 in S2 (Fig. 3). The baseline characteristics of the population are presented in Table 1.

The mean GA at diagnosis was $29.4 \pm 4$ weeks in S1 and $29.2 \pm 4$ weeks in S2. The proportion of FGR was 25 $\%(95 \% \mathrm{CI} 18$ - 32) in S1 and $59 \%(95 \%$ CI $53-65)$ in S2, $(\mathrm{p}<0.001)$. The median GA at birth for both strategies was 37 weeks (IQR 35 - 39).

There were no significant differences in the perinatal composite outcome between the two strategies (OR 0.99, 95\% CI 0.7 - 1.5, p 0.98) (Table 2), or in the subgroup analysis (early vs. late diagnosis) (Table 3 ). 
There were no differences in the global incidence of preterm birth (S1: $40 \%, 95 \%$ CI 33 - 48; S2: $33 \%, 95 \%$ CI 27 - 39; OR 0.76, 95\% CI 0.5 - 1.2, p 0.19) (Table 2). Subgroup analysis according to GA at diagnosis did not show differences either (Table 3).

In relation to complications of preterm birth, we found a significant reduction in the incidence of RDS in S2, both globally (OR $0.50,95 \%$ CI $0.3-0.9, \mathrm{p} 0.02$ ), and in the early diagnosis subgroup (OR $0.45,95 \%$ CI 0.2 $0.8, \mathrm{p} 0.01$ ) (Table 2, 3). In addition, we observed a significant reduction in the incidence of sepsis in S2, both globally (OR $0.30,95 \%$ CI $0.09-0.9, \mathrm{p} 0.04$ ) and in the early diagnosis subgroup (OR $0.25,95 \%$ CI $0.08-0.8$, p 0.02) (Table 2, 3).

We did not observe significant differences in the other prematurity-related complications, such as NEC (p 0.41) and ICH (p 1.00) (Table 2, 3).

\section{Discussion}

This study provides comparative data on the implementation of an expanded strategy for the prenatal management of small fetuses with respect to the traditional one, based exclusively on biometry and umbilical artery Doppler assessment. There were no differences in the perinatal composite outcome or preterm birth rate between both strategies. However, we found a reduction in RDS and sepsis in the cases managed with the expanded strategy.

Although the composite perinatal outcome rates found in this study were higher than in previously published studies, we attribute these differences to the outcome definition. The PORTO study [26] reported a composite perinatal adverse outcome of $5.2 \%, 7$ times lower than the present study, but this included more severe conditions such as death, intraventricular hemorrhage, periventricular leukomalacia, hypoxic-ischemic encephalopathy, necrotizing enterocolitis, bronchopulmonary dysplasia, and sepsis. The inclusion of NICU admission in our study resulted in a higher incidence of this composite outcome.

The group who initially described S2 published the results of a cohort of 1,197 pregnancies with low fetal weight, in which they reported a composite adverse outcome rate of 9.7\% [27]. Although this composite outcome consisted of the same variables as our study except for metabolic acidosis, they only included fetuses with late FGR, which are known to have a lower risk of perinatal complications. As our study also included early FGR, the global perinatal outcomes were worse, including a 2-3 times higher rate of preterm birth.

In our study, we did not observe significant differences in the preterm birth rate between both strategies. However, S2 showed a lower incidence of two complications related to prematurity, such as RDS and sepsis. This could be related to the fact that $\mathrm{S} 2$ provides more information about the fetal hemodynamic status, which could in turn translate into a better optimization of the moment of birth.

\section{Strengths and limitations}


To the best of our knowledge, this is the first study that compares the perinatal outcomes of two consecutive prenatal strategies for the management of small fetuses based on an updated definition of FGR, including both early and late cases. Another strength is that this study included a considerable number of pregnancies with low fetal weight, with a low percentage of loss to follow-up.

However, this study also has several limitations. Due to the study design, the results could have been influenced by a temporal bias related to potential changes in the neonatal protocols. Since it was an observational study, there may be unmeasured confounding influencing the results. Additionally, this study did not evaluate the possible effects of these interventions in the medium and long-term, such as cardio-metabolic changes and neurocognitive development.

\section{Conclusion}

The implementation of an expanded strategy for the management of small fetuses based on the inclusion of additional Doppler parameters did not show significant differences in the primary composite outcome or in the proportion of preterm birth when compared with a strategy based exclusively on fetal biometry and umbilical artery Doppler. However, the expanded strategy was associated with a lower incidence of RDS and neonatal sepsis, possibly related to an optimization in gestational age at birth according to risk.

The use of additional Doppler parameters such as AUt-PI, MCA-PI, and CPR included in an updated definition of FGR, together with a standardized management algorithm, may improve the detection of small fetuses with a higher risk of complications and consequently improve their perinatal outcomes.

Additional studies are warranted to validate these results in other populations.

Acknowledgments The authors would like to thank Diego Hernan Giunta, Cristina Maria Elizondo, Lucas Otaño, Pablo Hernan Brener, Mariana Leda Bucich, and Maria Lourdes Posadas-Martinez for their valuable contribution to this study.

Author contributions Conceptualization: Sabrina Paola Demirdjian, Adolfo Etchegaray and Cesar Hernan Meller; Methodology: Sabrina Paola Demirdjian and Adolfo Etchegaray; Acquisition of data: Sabrina Paola Demirdjian, Gonzalo Dosdoglirian, Maria Celeste Berruet. Formal analysis and investigation: Sabrina Paola Demirdjian, Adolfo Etchegaray and Cesar Hernan Meller; Writing - original draft preparation: Sabrina Paola Demirdjian; Writing - review and editing: Adolfo Etchegaray and Cesar Meller.

Funding The authors declare that no funds, grants, or other support were received during the preparation of this manuscript

\section{Declarations}

Conflict of interest The authors have no relevant financial or non-financial interests to disclose.

Ethics approval The study protocol was approved by our institutional review board. 
Consent for publication All authors agree on the final manuscript and the order of authors.

\section{References}

1. Practice Bulletin No. 134. Obstetrics \& Gynecology. 2013. pp. 1122-1133. doi:10.1097/01.aog.0000429658.85846.f9

2. McCowan LM, Figueras F, Anderson NH. Evidence-based national guidelines for the management of suspected fetal growth restriction: comparison, consensus, and controversy. American Journal of Obstetrics and Gynecology. 2018. pp. S855-S868. doi:10.1016/j.ajog.2017.12.004

3. Gordijn SJ, Beune IM, Thilaganathan B, Papageorghiou A, Baschat AA, Baker PN, et al. Consensus definition of fetal growth restriction: a Delphi procedure. Ultrasound Obstet Gynecol. 2016;48: 333-339. https://doi.org/10.1002/uog.15884

4. Lees CC, Stampalija T, Baschat A, da Silva Costa F, Ferrazzi E, Figueras F, et al. ISUOG Practice Guidelines: diagnosis and management of small-for-gestational-age fetus and fetal growth restriction. Ultrasound Obstet Gynecol. 2020;56: 298-312. DOI: 10.1002/uog.22134

5. Salam RA, Das JK, Bhutta ZA. Impact of intrauterine growth restriction on long-term health. Curr Opin Clin Nutr Metab Care. 2014;17: 249-254. DOI: 10.1097/MCO.0000000000000051

6. Kopec G, Shekhawat PS, Mhanna MJ. Prevalence of diabetes and obesity in association with prematurity and growth restriction. Diabetes Metab Syndr Obes. 2017;10: 285-295. DOI: 10.2147/DMSO.S115890

7. Ross MG, Beall MH. Adult Sequelae of Intrauterine Growth Restriction. Seminars in Perinatology. 2008. pp. 213-218. doi:10.1053/j.semperi.2007.11.005. DOI: 10.1053/j.semperi.2007.11.005

8. Hawkes N. Trial of Viagra for fetal growth restriction is halted after baby deaths. BMJ. 2018. p. k3247. doi:10.1136/bmj.k3247

9. Villanueva-García D, Mota-Rojas D, Hernández-González R, Sánchez-Aparicio P, Alonso-Spilsbury M, Trujillo-Ortega ME, et al. A systematic review of experimental and clinical studies of sildenafil citrate for intrauterine growth restriction and pre-term labour. Journal of Obstetrics and Gynaecology. 2007. pp. 255-259. doi:10.1080/01443610701194978

10. Sharp A, Cornforth C, Jackson R, Harrold J, Turner MA, Kenny LC, et al. Maternal sildenafil for severe fetal growth restriction (STRIDER): a multicentre, randomised, placebo-controlled, double-blind trial. Lancet Child Adolesc Health. 2018;2: 93-102. DOI:

$10.1016 / \mathrm{S} 2352-4642(17) 30173-6$

11. Spencer R, EVERREST Consortium, Ambler G, Brodszki J, Diemert A, Figueras F, et al. EVERREST 
prospective study: a 6-year prospective study to define the clinical and biological characteristics of pregnancies affected by severe early onset fetal growth restriction. BMC Pregnancy and Childbirth. 2017. doi:10.1186/s12884-017-1226-7

12. Say L, Metin Gülmezoglu A, Justus Hofmeyr G. Maternal oxygen administration for suspected impaired fetal growth. Cochrane Database of Systematic Reviews. 2003.

doi:10.1002/14651858.cd000137

13. Laurin J, Persson P-H. The effect of bedrest in hospital on fetal outcome in pregnancies complicated by intrauterine growth retardation. Acta Obstetricia et Gynecologica Scandinavica. 1987. pp. 407-411. doi:10.3109/00016348709022043

14. Say L, Metin Gülmezoglu A, Justus Hofmeyr G. Maternal nutrient supplementation for suspected impaired fetal growth. Cochrane Database of Systematic Reviews. 2003.

doi:10.1002/14651858.cd000148

15. Say L, Metin Gülmezoglu A, Justus Hofmeyr G. Betamimetics for suspected impaired fetal growth. Cochrane Database of Systematic Reviews. 2001. doi:10.1002/14651858.cd000036

16. Kingdom JCP, Burrell SJ, Kaufmann P. Pathology and clinical implications of abnormal umbilical artery Doppler waveforms. Ultrasound in Obstetrics and Gynecology. 1997. pp. 271-286. doi:10.1046/j.1469-0705.1997.09040271.x

17. Karsdorp VHM, van Vugt JMG, van Geijn HP, Kostense PJ, Arduim D, Montenegro N, et al. Clinical significance of absent or reversed end diastolic velocity waveforms in umbilical artery. The Lancet. 1994. pp. 1664-1668. doi:10.1016/s0140-6736(94)90457-x

18. Society for Maternal-Fetal Medicine Publications Committee, Berkley E, Chauhan SP, Abuhamad A. Doppler assessment of the fetus with intrauterine growth restriction. Am J Obstet Gynecol. 2012;206: 300-308. DOI: 10.1016/j.ajog.2012.01.022

19. Ott WJ. Intrauterine growth restriction and Doppler ultrasonography. Journal of ultrasound in medicine. 2000 Oct;19(10):661-5. DOI: 10.7863/jum.2000.19.10.661

20. Bamfo JE, Odibo AO. Diagnosis and management of fetal growth restriction. Journal of pregnancy. 2011 Oct;2011. DOI: 10.1155/2011/640715

21. Figueras F, Gardosi J. Intrauterine growth restriction: new concepts in antenatal surveillance, diagnosis, and management. American journal of obstetrics and gynecology. 2011 Apr 1;204(4):288-300. DOI: 10.1016/j.ajog.2010.08.055

22. Salomon LJ, Alfirevic Z, Da Silva Costa F, Deter RL, Figueras F, Ghi T, et al. ISUOG Practice Guidelines: ultrasound assessment of fetal biometry and growth. Ultrasound Obstet Gynecol. 2019;53: 715-723. DOI: $10.1002 /$ uog.20272 
23. Melamed N, Baschat A, Yinon Y, Athanasiadis A, Mecacci F, Figueras F, et al. FIGO (international Federation of Gynecology and obstetrics) initiative on fetal growth: best practice advice for screening, diagnosis, and management of fetal growth restriction. Int J Gynaecol Obstet. 2021;152 Suppl 1: 3-57. https://doi.org/10.1002/ijgo.13522

24. Figueras F, Gratacos E. Stage-based approach to the management of fetal growth restriction. Prenat Diagn. 2014;34: 655-659. DOI: 10.1002/pd.4412

25. Savchev S, Figueras F, Sanz-Cortes M, Cruz-Lemini M, Triunfo S, Botet F, et al. Evaluation of an optimal gestational age cut-off for the definition of early- and late-onset fetal growth restriction. Fetal Diagn Ther. 2014;36: 99-105. DOI: 10.1159/000355525

26. Unterscheider J, Daly S, Geary MP, Kennelly MM, McAuliffe FM, O’Donoghue K, et al. Optimizing the definition of intrauterine growth restriction: the multicenter prospective PORTO Study. Am J Obstet Gynecol. 2013;208: 290.e1-6. DOI: 10.1016/j.ajog.2013.02.007

27. Meler E, Mazarico E, Eixarch E, Gonzalez A, Peguero A, Martinez J, et al. A 10-year experience of protocol-based management of fetal growth restriction: perinatal outcomes in late pregnancy cases diagnosed after 32 weeks. Ultrasound Obstet Gynecol. 2020. doi:10.1002/uog.23537

\section{Figures}

Fig. 1 Classification and management recommended by Strategy 1

EFW: Estimated fetal weight. UmA-PI: Umbilical artery pulsatility index. SGA: Small for gestational age. FGR: Fetal growth restriction. >p95: greater than the 95th percentile. EDF: end-diastolic flow.

Fig. 2 Classification and management recommended by Strategy 2

EFW: Estimated fetal weight. UmA-PI: Umbilical artery pulsatility index. MCA-PI: Middle cerebral artery pulsatility index. CPR: cerebro-placental ratio. UtA-PI: mean pulsatility index of the uterine arteries. DV: Ductus venosus. DV-PI: Ductus venosus pulsatility index. $>$ p95: greater than the 95th percentile. EDF: end-diastolic flow. SGA: small for gestational age. FGR: Fetal Growth Restriction.

Fig. 3 Flow-chart

\section{Tables}


Table 1 Baseline characteristics of the population

$\mathrm{SD}=$ Standard Deviation. $\mathrm{BMI}=$ Body Mass Index. GDM= Gestational diabetes. FGR= Fetal Growth

Restriction. Early diagnosis $=$ Diagnosis of low fetal weight $\leq 32$ weeks. Late diagnosis= Diagnosis of low fetal weight $>32$ weeks. IQR= Interquartile range. gr= grams.

Table 2 Comparison of primary and secondary outcomes according to strategy

Perinatal composite outcome $=$ Considered positive when at least one of the following events occurred: NMV, need for NICU, or perinatal death. S1= Strategy 1, S2= Strategy 2. OR= odds ratio comparing Strategy 2 with 1. 95\% CI: 95\% confidence interval. NMV= need for mechanical ventilation. NICU= Neonatal Intensive Care Unit. $\mathrm{RDS}=$ Respiratory distress syndrome. $\mathrm{NEC}=$ necrotizing enterocolitis. $\mathrm{IVH}=$ intraventricular hemorrhage. The results are expressed with absolute and relative frequencies (\%). NS= Not statistically significant.

Table 3 Comparison of primary and secondary outcomes according to early and late diagnosis.

Perinatal composite outcome $=$ Considered positive when at least one of the following events occurred: NMV, need for NICU, or perinatal death. Early diagnosis= Diagnosis of low fetal weight $\leq 32$ weeks $(\mathrm{S} 1 \mathrm{n}=119$, S2 $\mathrm{n}=196)$, Late diagnosis $=$ Diagnosis of low fetal weight $>32$ weeks $(\mathrm{S} 1 \mathrm{n}=44, \mathrm{~S} 2 \mathrm{n}=37) . \mathrm{S} 1=$ Strategy $1, \mathrm{~S} 2=$ Strategy 2. $\mathrm{OR}=$ odds ratio comparing Strategy 2 with $1.95 \% \mathrm{CI}=95 \%$ confidence interval. $\mathrm{RDS}=$ Respiratory distress syndrome. $\mathrm{NEC}=$ necrotizing enterocolitis. $\mathrm{IVH}=$ intraventricular hemorrhage. The results are expressed with absolute and relative frequencies $(\%)$. NS= Not statistically significant. 
Figures

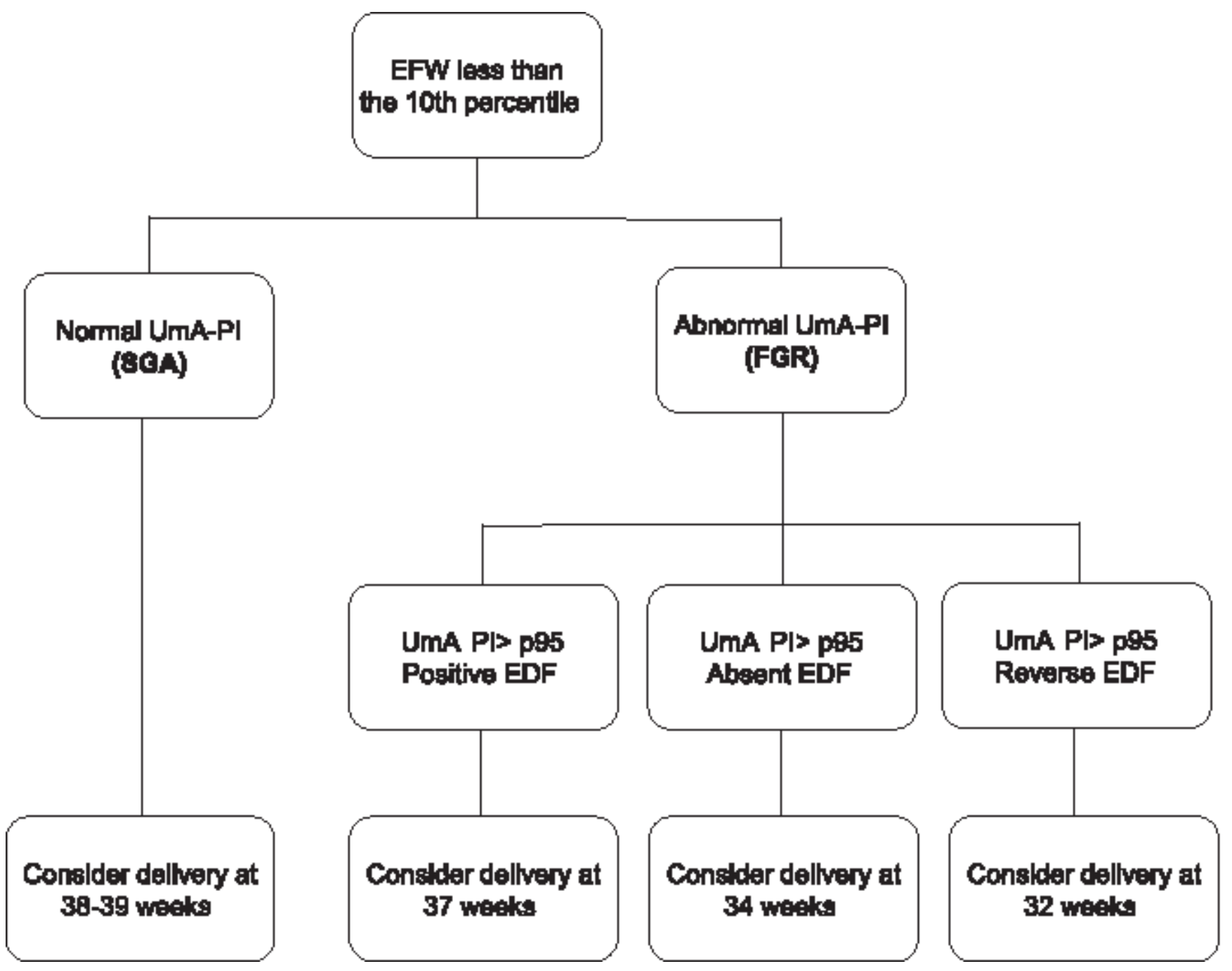

Figure 1

Classification and management recommended by Strategy 1 EFW: Estimated fetal weight. UmA-PI: Umbilical artery pulsatility index. SGA: Small for gestational age. FGR: Fetal growth restriction. $>$ p95: greater than the 95th percentile. EDF: end-diastolic flow. 


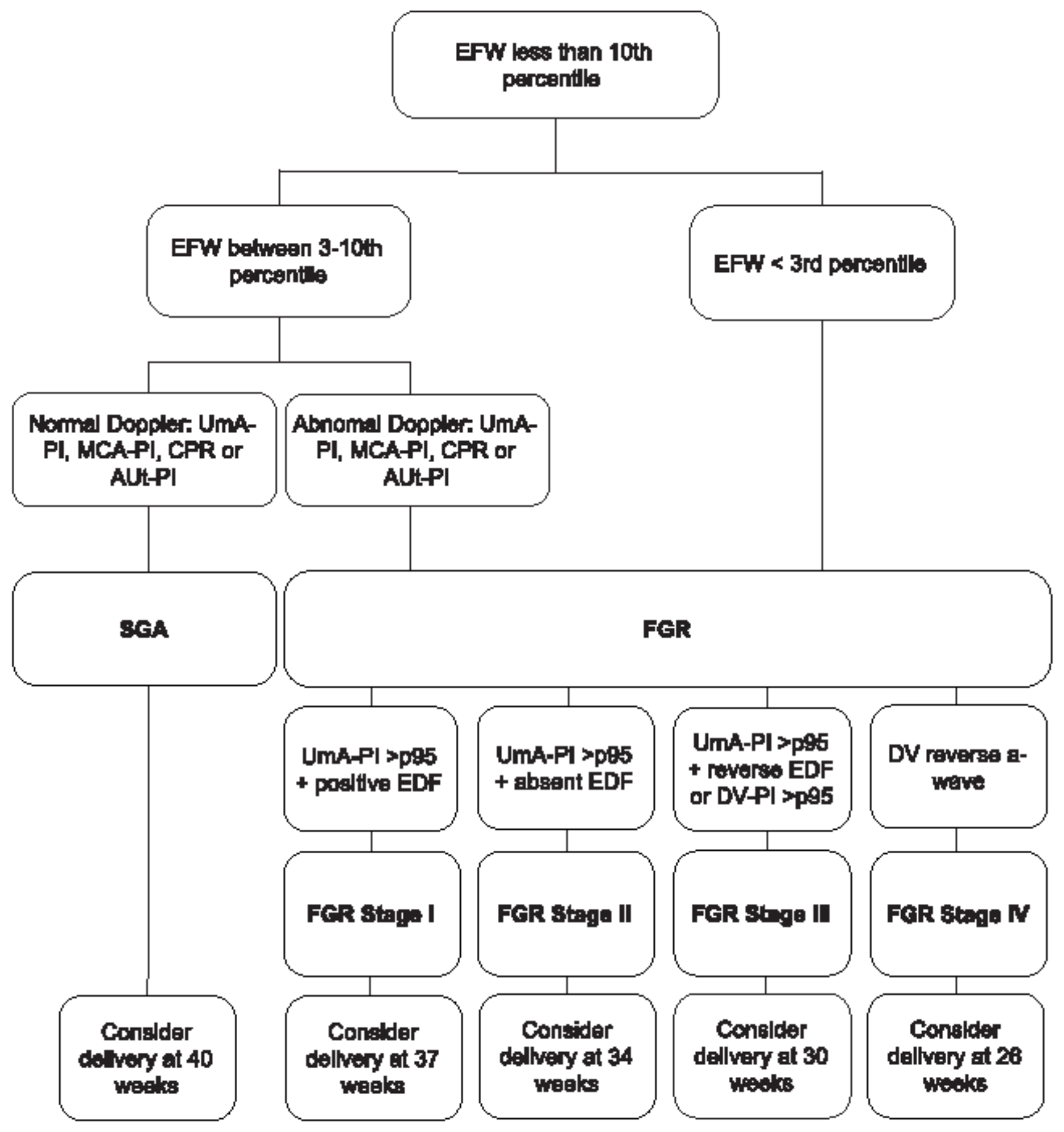

Figure 2

Classification and management recommended by Strategy 2 EFW: Estimated fetal weight. UmA-PI: Umbilical artery pulsatility index. MCA-PI: Middle cerebral artery pulsatility index. CPR: cerebro-placental ratio. UtA-PI: mean pulsatility index of the uterine arteries. DV: Ductus venosus. DV-PI: Ductus venosus pulsatility index. > p95: greater than the 95th percentile. EDF: end-diastolic flow. SGA: small for gestational age. FGR: Fetal Growth Restriction. 


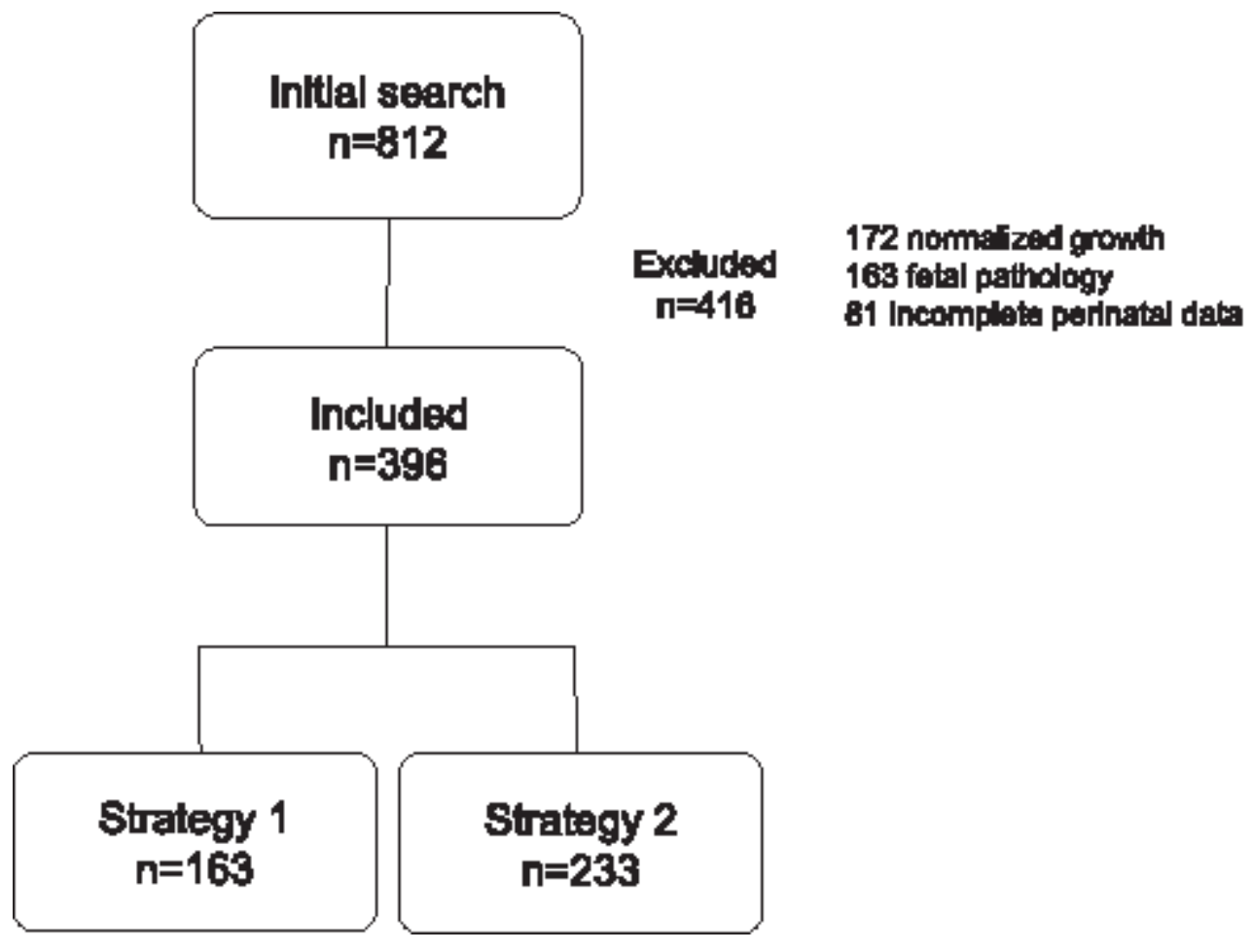

Figure 3

Flow-chart

\section{Supplementary Files}

This is a list of supplementary files associated with this preprint. Click to download.

- Table1.pdf

- Table2.pdf

- Table3.pdf 